\title{
Streamers and adjacent regions observed by UVCS/SOHO: A comparison between different phases of solar activity
}

\author{
R. Ventura ${ }^{1}$, D. Spadaro ${ }^{1}$, G. Cimino ${ }^{1}$, and M. Romoli ${ }^{2}$ \\ ${ }^{1}$ INAF - Osservatorio Astrofisico di Catania, via S. Sofia 78, 95123 Catania, Italy \\ e-mail: rve@ct.astro.it \\ ${ }^{2}$ Dipartimento di Astronomia e Scienza dello Spazio, Università di Firenze, L.go E. Fermi 2, 50125 Firenze, Italy
}

Received 2 August 2004 / Accepted 20 September 2004

\begin{abstract}
UVCS/SOHO observations of the O VI resonance doublet and HI Ly $\alpha$ line intensities and profiles, together with measurements of the visible linearly polarized radiance, have been performed during two MEDOC campaigns in 1997 and 2000 , i.e. near solar minimum and approaching the solar maximum phase, respectively. During both observational runs midlatitude coronal regions in the West limb of the Sun have been scanned over a range of heliocentric distance from 1.39 to 4.1 $R_{\odot}$, to study the plasma properties of streamers and adjacent regions, such as ion kinetic temperature, electron density and outflow velocity, paying particular attention to comparing plasma conditions deduced for different ions in coronal structures observed on different days and during different phases of solar activity. Besides confirming some previous findings on significant differences between open and closed field-line structures at solar minimum, our results provide some evidence for differences in kinetic temperature among mid-latitude solar minimum streamers observed on different days from about $2 R_{\odot}$ outwards, as well as in their dynamical conditions at heliocentric distances greater than $3.6 R_{\odot}$. For observations carried out in 2000, conversely, the mid-latitude coronal streamers and their surroundings are about 3 times, and more than one order of magnitude brighter, respectively, than their solar minimum counterparts and exhibit very similar kinetic and dynamical conditions. The kinetic temperatures in adjacent regions are higher than in streamers (by about a factor of 2) only within $2 R_{\odot}$, while at greater heights such differences vanish, making it difficult to discriminate between open and closed structures. This is opposite to the behaviour detected at solar minimum, when adjacent regions appear to be characterized by kinetic temperatures progressively higher and higher than in streamers with increasing height, from $2 R_{\odot}$ outwards. Therefore, a clear characterization of open and closed configurations near the solar maximum might be quite difficult, probably due both to the intrisically more complex magnetic configuration of the corona in this phase of the solar activity and the line-of-sight contamination effects that in a highly structured solar corona may strongly mix background and foreground plasma with different properties. The transition from the solar minimum to maximum also seems characterized by a global increase in the electron density inside streamers of about a factor of 4 at $1.7 R_{\odot}$ and then it progressively decreases with height.
\end{abstract}

Key words. Sun: corona - Sun: solar wind - Sun: UV radiation

\section{Introduction}

In recent years the dynamics of the solar wind and heating mechanisms at work in the coronal plasma have been extensively investigated. Space-based observations from Yohkoh, Ulysses, ACE (Advanced Composition Explorer), TRACE (Transition Region and Coronal Explorer) and SOHO (SOlar and Heliospheric Observatory) have greatly improved our understanding of the physical state of the coronal plasma in regions of different magnetic configuration and during different phases of the solar activity cycle. At present it seems well established that the two solar wind regimes, the high- and slowspeed wind, originate from different magnetic topologies. The relatively steady fast wind flows from coronal holes, large-scale open magnetic structures, which at solar minimum widen out at polar caps and persist over a large fraction of the activity cycle.
The much more variable slow wind seems to be associated with closed magnetic configurations, the coronal streamers, which at the solar minimum - appear to be confined at low latitudes around the equatorial belt, clearly emerging from a dark background corona. Approaching solar maximum, the large polar coronal holes shrink, fragment and eventually disappear, while small-scale and relatively short-lived coronal holes form at low and mid latitudes, and many streamers appear ubiquitously in a diffusely bright corona. As a consequence of the drastic topological modification in the global coronal magnetic field, the solar wind structure changes significantly: fast high-latitude wind disappears and a much more complex and highly structured mixture of flows in a wide range of speeds originates from a variety of sources (small coronal holes, active regions, coronal mass ejections and streamers) at all latitudes (see, e.g., McComas et al. 2002a,b; Smith et al. 2003). 
However, many puzzling and still well debated aspects of this understood global picture need to be investigated deeply in order to get additional constraints for the development of coronal heating and wind acceleration models. For instance, not much is known about where, within coronal holes and streamers, the solar wind actually originates, as well as how and where, in terms of both radial distance from the Sun and location in coronal sub-structure, the transition from slow to fast solar wind takes place. Many recent works focused on these topics, but general conclusive evidence has not yet been found (e.g. Noci et al. 1997; Sheeley et al. 1997; Habbal et al. 1997; Raymond et al. 1997; Hassler et al. 1999; Ofman 2000; Banerjee et al. 2000; Giordano et al. 2000; Patsourakos \& Vial 2000; Strachan et al. 2002; Teriaca et al. 2003; Gabriel et al. 2003). On the other hand, only a few similar studies have been based on observations carried out during the rising phase of the solar cycle and at its maximum (see, e.g., McComas et al. 2002b).

A substantial contribution in this context may come from detailed observations of coronal regions where the solar wind originates over a range of heliocentric distances as large as possible in order to obtain accurate profiles of the most relevant wind physical parameters such as temperature, density, velocity and elemental abundance. The Ultraviolet Coronagraph Spectrometer (UVCS, Kohl et al. 1995) on board SOHO (Domingo et al. 1995), offering the opportunity to probe the dynamics of the extended solar corona up to $10 R_{\odot}$ through the observation of the most intense EUV emission line profiles, can be considered one of the most suitable instruments to accomplish this task. Many recent works, based on observations carried out by UVCS, focused on the latitudinal behaviour of the solar wind properties at the interface between streamers and coronal holes at low heliocentric distances during the minimum phase of solar activity (Abbo \& Antonucci 2002; Antonucci et al. 1997; Dobrzycka et al. 1999; Strachan et al. 2000; Zangrilli et al. 1999, 2002). More systematic sequences of very detailed latitudinal scans at several heliocentric distances, covering the range 1.4-2.5 $R_{\odot}$, are those reported by Spadaro et al. (2004), who observed different streamers near solar mimimum to investigate the variations of wind parameters across the streamer boundaries.

However, there is still a lack of similar papers addressing either the radial and latitudinal behaviour of physical parameters in the low corona during solar maximum or its possible dependence on solar cycle. Here we report the results obtained from data collected by UVCS /SOHO during MEDOC (Multi-Experiment Data Operations Center for SOHO) campaigns \# 2 and \# 6, both coordinated at the Institute d'Astrophysique Spatiale in Orsay-Paris and held respectively on October-November, 1997, when the Sun was close to the minimum of its activity, and on October-November, 2000, near the solar maximum. UVCS/SOHO supported these campaigns with specially designed observations and synoptic sequences. The main purpose of the dedicated observations was to study the extended solar corona inside and immediately outside midlatitude streamers, in order to characterize plasma properties in the two regions. The two sets of data acquired during two different phases of the solar activity cycle also gave us the opportunity to investigate the possible dependence of the physical conditions inside and outside streamers on solar activity. This paper is organized as follows: the observational details and data analysis are reported in Sect. 2, the relevant results are presented and briefly discussed in Sect. 3, while in Sect. 4 we draw some conclusions.

\section{Observations and data reduction}

The UVCS consists of two UV channels, one working in the range 1145-1287 $\AA$ and the other one operating between 984-1080 $\AA$, optimized respectively for observations in HI $\operatorname{Ly} \alpha$ and O VI resonance doublet lines, and a whitelight channel (WLC) which allows us to measure the linearly polarized radiance brightness $(\mathrm{pB})$ of the K-corona in the 4500-6000 A band. The three channels simultaneously observe the same portion of the solar corona in the range of heliocentric distance $1.5-10 R_{\odot}$. Intensities and profiles of strong EUV emission lines, such as $\mathrm{HI} \operatorname{Ly} \alpha$ and $\mathrm{Ly} \beta$ and the O VI resonance doublet at $1032 \AA$ and $1037 \AA$, together with some other minor ion lines, can be measured.

The present work is based on two data sets collected from November 3 to 9, 1997, during MEDOC campaign \# 2, and from October 30 to November 5, 2000, during MEDOC campaign \# 6. Radial scans of the corona above the West limb of the Sun were carried out during both campaigns, selecting a different position angle (PA, measured counterclockwise from the North Pole) daily. The spectrometer slit scanned the solar corona from $1.39 R_{\odot}$ to $4.12 R_{\odot}$ in the $250^{\circ}-300^{\circ} \mathrm{PA}$ range in 1997, and analogously from $1.39 R_{\odot}$ to $3.09 R_{\odot}$ in the sector $240^{\circ}-300^{\circ}$ in 2000 . Moreover on November 1,2000 a very dense radial scan was performed.

During the first 4 days of MEDOC campaign \# 2, the midlatitude streamer region located in the North-West quadrant and overlying a relatively faint chromospheric active region was monitored and its temporal evolution followed. The last day was devoted to observing two mid-latitude streamers located in the South-West quadrant. During MEDOC campaign \# 6 , we observed two different mid-latitude streamer regions, one over an interval of 3 days and the other for one day, both associated with highly structured active region complexes. The streamer observed on Nov. 5, 2000 was flanked by an equatorial hole (see Fig. 1), clearly seen standing out against the solar disk and approaching the solar limb in the SOHO/EIT (Delaboudinière et al. 1995) images of previous days. Details of the observation sequences concerning both campaigns are reported in Table 1. Figure 1 shows, as an example of the daily radial scans, the instantaneous positions of the UVCS slit superimposed on white-light images of the corona taken in the range 2-6 $R_{\odot}$ by the SOHO/LASCO C2 coronagraph (Large Angle Spectroscopic Coronagraph, Brueckner et al. 1995), on November 9, 1997 and November 5, 2000.

During MEDOC campaign \# 2, intensities and profiles of the HI Ly $\alpha$ and OVI resonance doublet were acquired using both the Ly $\alpha$ and the OVI spectrometer channels; during MEDOC campaign \# 6, only the O VI channel was available for observations and our data set is restricted to O VI intensities and profiles. The data have been taken with a 

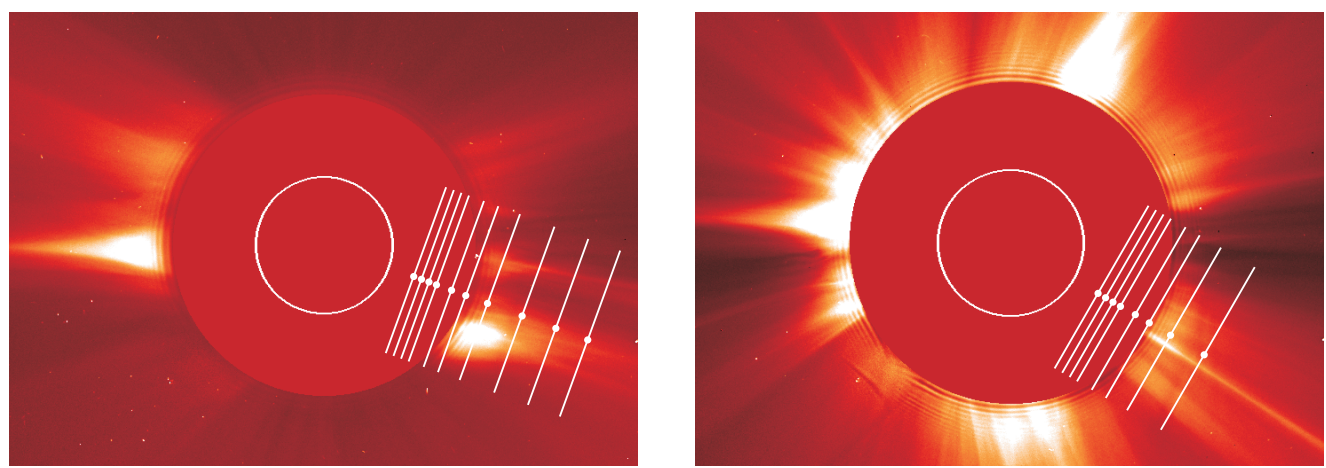

Fig. 1. LASCO C2 white-light images of the solar corona taken on November 9, 1997 (left panel) and November 5, 2000 (right panel). The instantaneous positions of the O VI channel slit of UVCS (white segments) during the radial scans are shown superimposed on the LASCO images. The white dots mark the positions along the slit closest to the solar limb, and to which the heliocentric distances and PA reported in Table 1 refer. It is interesting to note the deep difference in the appearence of the corona in the two LASCO images taken at the mimimum (left panel) and at maximum (right panel) of the solar activity cycle. The solar mimimum-streamer definitely emerges from a very dark background while the streamer observed in 2000 (flanked by an equatorial hole) is only one of the various and very bright streamers present at all latitudes in a solar corona looking uniformly bright.

Table 1. Log of the observing sequences.

\begin{tabular}{|c|c|c|c|}
\hline & Date & $\begin{array}{c}\mathrm{PA} \\
\text { (degrees) }\end{array}$ & $\begin{array}{c}\text { Heliocentric distance } \\
\qquad\left(r / R_{\odot}\right)\end{array}$ \\
\hline \multicolumn{4}{|l|}{1997} \\
\hline & Nov. 3 & 300 & $1.39,1.51,1.63,1.75,1.98,2.21,2.55,3.09,3.62,4.12$ \\
\hline & Nov. 5 & 290 & " \\
\hline & Nov. 6 & 270 & $"$ \\
\hline & Nov. 7 & 285 & " \\
\hline & Nov. 9 & 250 & $"$ \\
\hline \multicolumn{4}{|l|}{2000} \\
\hline & Oct. 30 & 300 & $1.39,1.51,1.63,1.75,1.98,2.21,2.55,3.09$ \\
\hline & Oct. 31 & 290 & " \\
\hline & Nov. 1 & 300 & $1.39,1.45,1.51,1.63,1.75,1.87,1.98,2.10,2.21,2.55,3.09,3.62$ \\
\hline & Nov. 5 & 240 & $1.39,1.51,1.63,1.75,1.98,2.21,2.55,3.09$ \\
\hline
\end{tabular}

spatial binning of 6 pixels giving a spatial resolution of $42 \operatorname{arcsec}(7 \operatorname{arcsec}$ for each pixel) over a slit 40 arcmin long. The slit width was $50 \mu \mathrm{m}$ in both channels up to $2.0 R_{\odot}$, while in the O VI channel it was increased to $200 \mu \mathrm{m}$ (1997 data) and $150 \mu \mathrm{m}$ (2000 data) at higher heliocentric distances to optimize both spectral resolution and photon flux. The spectral resolution was about $0.3 \AA$ at any heliocentric distance in the Ly $\alpha$ channel; in the O VI channel it was about $0.2 \AA$ up to $2.0 R_{\odot}$, and $\sim 0.8 \AA$ (1997 data) and $\sim 0.6 \AA$ (2000 data) at higher heliocentric distances. Simultaneous $\mathrm{pB}$ measurements were also performed both in 1997 and 2000 with the WLC over a $14 \operatorname{arcsec} \times 14$ arcsec area located approximately at the center of the UVCS instantaneous field of view (Romoli et al. 1997).

The most recent version of the UVCS Data Analysis and processing Software (DAS, originally developed by C. Benna, A. van Ballegoijen, J. Raymond and S. Giordano) was used for flat-field correction, wavelength and radiometric calibration, removal of image distorsion and spectra extraction (Gardner et al. 1996; Kohl et al. 1997). Panasyuk's package (available from the Harvard-Smithsonian Center For Astrophysics website: cfa-www.harvard.edu/uvcs) was used to remove the disk-scattered stray light contribution to both the H I Ly $\alpha$ and
O VI doublet lines, and the contribution from the interplanetary scattered Ly $\alpha$ line contained in the observed Ly $\alpha$ profile. Subtraction of the background and instrumental broadening correction were also performed before single Gaussian fits to the line profiles were obtained, in order to determine their intensity and $1 / e$ half width.

To study possible differences in the physical parameters characterizing streamers and adjacent regions, we first localized, for each exposure and examined line, the streamer borders by using the 1/e intensity criterion (see, e.g., Abbo \& Antonucci 2002); such borders are shown in Fig. 2, as an example. Then we extracted the spectra relevant to both the observed streamer and a strip approximately 10-15 arcmin wide, adjacent to the streamer leg, further binning the original data over the appropriate spatial ranges along the slit (see Fig. 2).

\section{Results and discussion}

\subsection{Line intensities}

Figure 3 reports the total intensities of the O VI $1032 \AA$ and Ly $\alpha$ lines as a function of heliocentric distance for the regions 


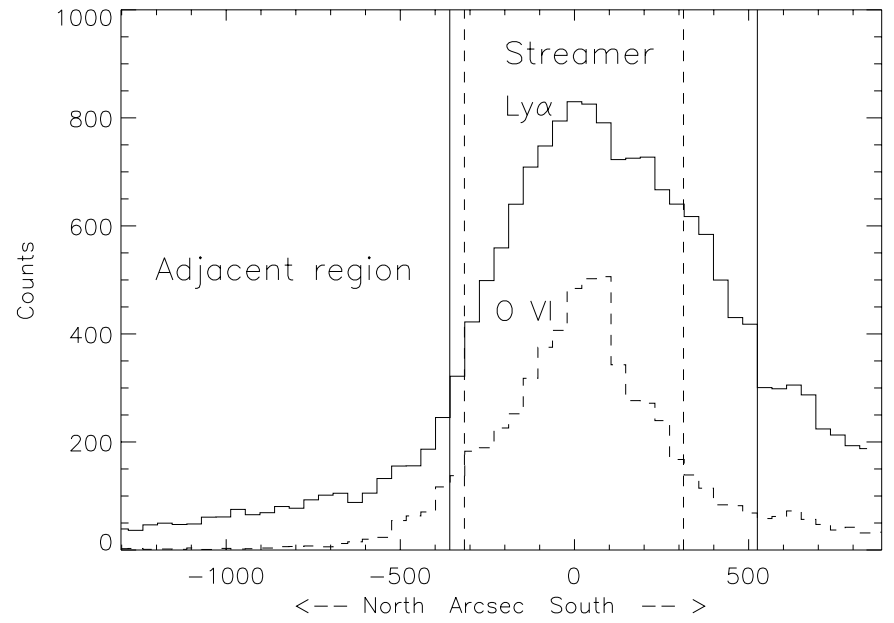

Fig. 2. Ly $\alpha$ (solid line) and O VI $1032 \AA$ (dashed line) line brightness profiles along the UVCS slit at $1.39 R_{\odot}$ and $\mathrm{PA}=300^{\circ}$ observed on Nov. 3, 1997. Solid and dashed vertical lines mark off the "streamer" and "adjacent region" bands, respectively, for $\operatorname{Ly} \alpha$ and O VI, selected to extract the relevant spectra.

inside mid-latitude streamers and adjacent to them, observed in 1997 and 2000. Since in only a few cases the streamer axes coincide with the slit center at each value of heliocentric distance and PA reported in Table 1, and different points along the slit correspond to different heights above the solar limb, the effective position along the slit of the streamer axis and of the adjacent region center was converted to height by straightforward geometric computations. The heliocentric distance values reported in Fig. 3 refer for each point to these effective coordinates. On Nov. 9, 1997, two large streamers, partially superimposed on the line-of sight, filled completely the instantaneous UVCS field of view already at low heliocentric distances, preventing us from isolating an adjacent band and extracting the relevant spectra at any point of the radial scan. The data referring to these streamers are reported in Fig. 3 by different symbols. Analogously, Fig. 3 reports only the intensities relevant to the observed streamer whenever the structure, progressively fanning out with heliocentric distance, covers the whole UVCS slit field of view. This occurred in some radial scans performed in 2000. Since Ly $\alpha$-streamers are on average a factor of 7 brighter than their O VI counterparts, the Ly $\alpha$ intensities in Fig. 3 have been scaled by an appropriate factor for an easier comparison with the O VI $1032 \AA$ intensity curves. We computed the errors affecting the data points in Fig. 3 as the standard deviation $( \pm 1 \sigma)$ of the Poissonian photon count statistics; the corresponding error bars are not plotted because they are smaller than the symbol size.

The Ly $\alpha$ and O VI $1032 \AA$ total intensity radial profiles referring to the streamers observed in 1997 show a different behaviour: the Ly $\alpha$ falls off with height by little more than about 2 orders of magnitude in comparison with a quicker decrease by more than about 3 orders of magnitude for the O VI $1032 \AA$ line. This mainly reflects the different dependence on density of the two lines, and in addition, the different sensitivity of the relevant radiative components to the outflow velocity through the Doppler dimming effect (see, e.g.,
Hyder \& Lites 1970; Beckers \& Chipman 1974; Noci et al. 1987). Streamers observed on different days in 1997 show very similar total intensities both in Ly $\alpha$ and O VI lines: the $\mathrm{O}$ VI in-streamer intensities almost overlap within $1.8 R_{\odot}$, while at higher heliocentric distances the different profiles slightly spread out, differing by about a factor of 2 , at most (at $4 R_{\odot}$ ). The Ly $\alpha$ in-streamer intensities exhibit a similar behaviour but the different curves begin to diverge at higher heliocentric distances $\left(\geq 3 R_{\odot}\right)$. This small divergence reflects the slightly different fall with height over the solar limb of the density characterizing the in-streamer regions scanned during the observational week (see also Sect. 3.4), and implies that the brightness of the streamer observed from Nov. 3 to Nov. 7 did not change significantly over the observational period of 4 days.

Both the Ly $\alpha$ and O VI $1032 \AA$ total intensity profiles referring to the out-of-streamer regions observed in 1997 show well marked differences in comparison to their in-streamer counterparts. In both lines the less dense adjacent regions appear systematically fainter at any heliocentric distance, the relevant intensities being a factor of about one third less intense up to about $2.0 R_{\odot}$, and going down to $1 / 5$ in $\mathrm{O}$ VI and $1 / 6$ in $\operatorname{Ly} \alpha$, at $4 R_{\odot}$. These increasing differences between in- and out-of-streamer region intensities, occurring with increasing height, could be most likely ascribed to a progressively increasing Doppler dimming effect due to the different dynamical conditions of the regions flanking streamers where the solar wind is expected to progressively accelerate with height (see also Sect. 3.3). However, the streamer-adjacent region contrast could also depend on the $1 / e$ criterion adopted to define the border between the two regions or the spatial profile of a streamer which may change from sharply peaked at low heliocentric distances to flat-topped at greater heights, and therefore basically on geometrical projection effects.

The intensity profiles referring to the adjacent regions observed in different days are very similar to each other in both lines, except that inferred for the O VI line on Nov. 7, 1997, which appears systematically brighter, and that for the Ly $\alpha$ line on Nov. 3, 1997, which appears systematically darker.

The O VI total intensity profiles observed in 2000 show a different behaviour. The individual intensity radial profiles relevant to streamers are indistinguishable from each other at any heliocentric distance. Analogously, the intensity profiles characterizing the regions surrounding the streamers cluster in a very narrow strip located about $50 \%$ lower than the corresponding streamer intensity curves.

In order to characterize more quantitatively the transition from solar minimum to solar maximum we derived also mean intensity radial profiles representative of streamers and adjacent regions in 1997 and 2000, respectively, by averaging the corresponding individual curves. Then, for the two kinds of examined regions, we divided the radial profile derived for 2000 by that derived for 1997 . The results are shown in the rightbottom panel of Fig. 3. The streamers observed in 2000 are on average from 2 up to 4 times brighter than those observed at solar minimum, with the greatest difference between 1.6 and $2.5 R_{\odot}$. The regions surrounding solar maximum and solar minimum streamers show even more pronounced differences: at $1.4 R_{\odot}$ the regions adjacent to solar maximum streamers are 


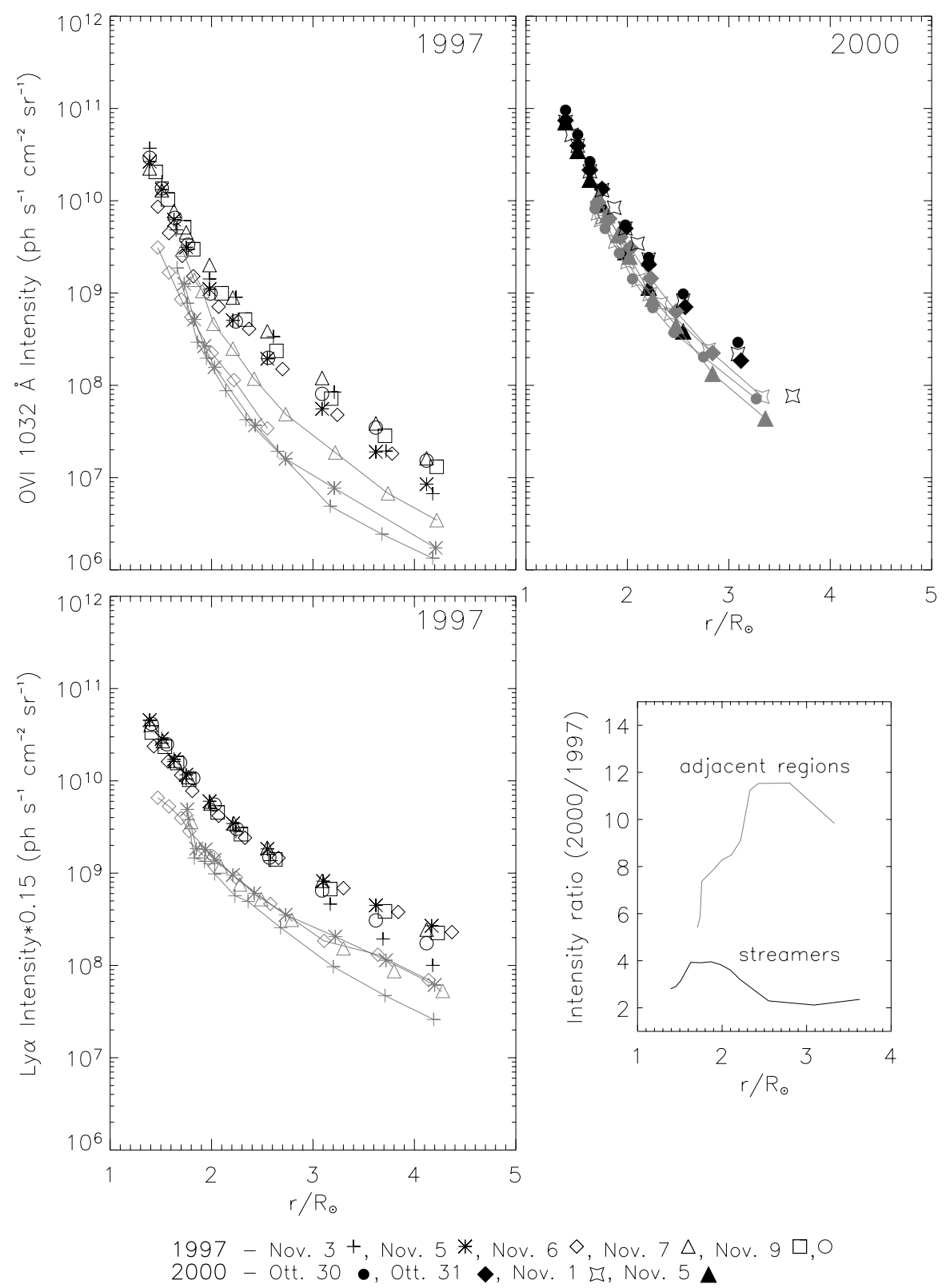

Fig. 3. O VI $1032 \AA$ (top) and Ly $\alpha$ (bottom-left) line-integrated intensities referring to the mid-latitude streamer regions observed in 1997 and 2000 , reported as a function of heliocentric distance. Different symbols refer to streamers (black points) and adjacent regions (grey points connected by grey lines) observed on different days according to the labels reported at the bottom. Bottom-right panel: 2000/1997 O vi 1032 ̊ intensity ratios for streamers (black line) and adjacent regions (grey line), obtained averaging the individual intensity profiles for the relevant structures observed during the two MEDOC campaigns.

about 5 times brighter than their solar minimum counterparts. The brightness ratio increases with increasing heliocentric distance, reaching a maximum between 2.4 and $2.8 R_{\odot}$, where the solar maximum adjacent regions are more than one order of magnitude brighter than similar regions observed in 1997; then it decreases at higher heliocentric distances. This behaviour might be attributed to a real global enhancement in the emissivity level of the solar corona outside streamers, due to an effective increase in density on open field lines from solar minimum to solar maximum, which, in turn, should determine a less marked streamer-adjacent region contrast, as it is evident also in the LASCO-C2 white-light images shown in Fig. 1. On the other hand, many unresolved small streamers superimposed along the line-of-sight that can enhance the emission in regions adjacent to streamers might mimic the same result. Our data do not allow us to discriminate between the two hypotheses.

\subsection{Kinetic temperature}

The broadening of a spectral line depends on the velocity distribution of the particles (atoms, ions or electrons) emitting or scattering the observed radiation along the line of sight, i.e. mainly perpendicular to the magnetic field, and can be 


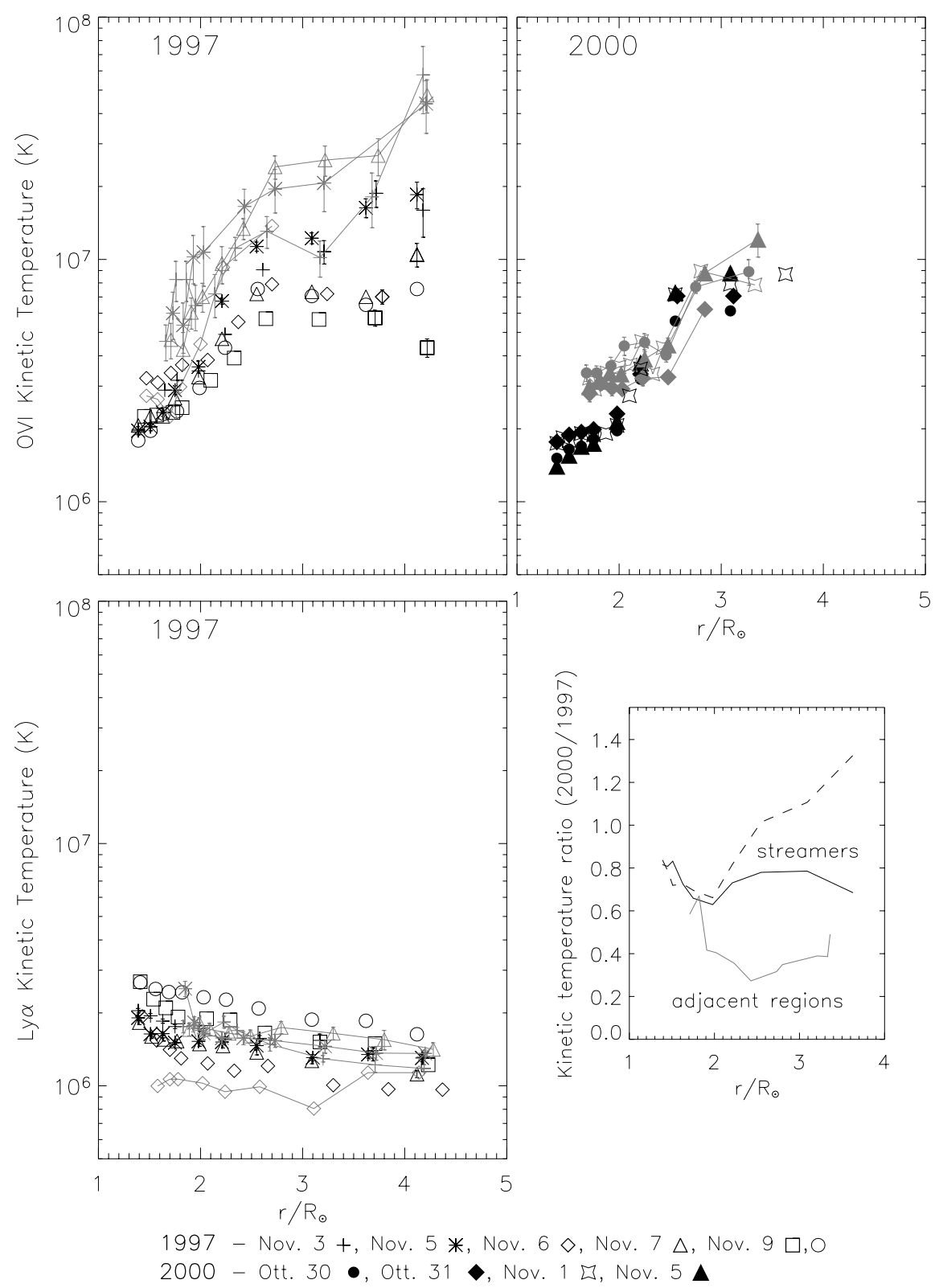

Fig. 4. As in Fig. 3, for the O VI and Ly $\alpha$ kinetic temperatures. Bottom-right panel: 2000/1997 kinetic temperature ratios referring to O VI streamers (black solid and dashed lines) and adjacent regions (grey line), respectively, obtained by averaging the individual profiles of the relevant structures. Two different curves referring to the streamers are reported; they result from including (solid line) or not (dashed line) the radial profiles inferred for the observations carried out on Nov. 3 and 5 in the 1997 mean curve.

expressed in terms of the plasma perpendicular kinetic temperature. This quantity measures, in addition to thermal motions, also non-thermal effects such as those induced by waves propagating, damping and dissipating in the coronal plasma. For this reason the kinetic temperature is to be considered one of the crucial parameters to investigate the coronal heating mechanisms and energy deposition processes related to solar wind acceleration (see, e.g., Kohl et al. 1998).

Figure 4 shows the radial profiles deduced from the spectral line $1 / e$ half widths of the O VI $1032 \AA$ and Ly $\alpha$ line perpendicular kinetic temperature, referring to the regions inside and adjacent to the mid-latitude streamers observed both in 1997 and 2000. The kinetic temperature uncertainties have been estimated from the photon counting statistics affecting both the examined line intensities and spectral line profiles, here assumed Gaussian, through the error propagation statistical treatment. The corresponding $\sigma$ values are plotted as error bars in Fig. 4 whenever greater than the symbol size.

The most evident feature characterizing the OVI and Ly $\alpha$ kinetic temperature radial profiles obtained in 1997 is their opposite trend: the OVI kinetic temperature increases, more quickly in regions adjacent to streamers than in the streamers themselves, while the $\operatorname{Ly} \alpha$ one slightly decreases towards high heliocentric distances with a similar behaviour, on average, in both regions. More in detail, at low heliocentric distances the $\mathrm{O}$ VI and Ly $\alpha$ kinetic temperatures of streamer regions appear to be comparable to each other, then, from $2.0 R_{\odot}$ outwards, the OVI kinetic temperatures become larger and larger with 
increasing height, until it exceeds the HI one by more than a factor of 10 at $4 R_{\odot}$. This result is in good agreement with that found by Strachan et al. (2002) in their analysis along the axis of an equatorial streamer observed on April 1997 and by Frazin et al. (2003), who analyzed the kinetic behaviour of an equatorial streamer monitored on October 1996. Some additional information comes from the analysis of the temporal evolution of the streamer region observed over an interval of 4 days and, also, from the comparison between the behaviour of regions at different latitudes observed on different days. The streamer region observed during the first 4 days of the MEDOC campaign \# 2 is particularly interesting; we note that from about $1.98 R_{\odot}$ outwards the O VI line seems to evolve, exhibiting substantially different kinetic properties: on Nov. 3 and 5, 1997, the enhacement in kinetic temperature abruptly occurs within a few tenths of a solar radius, although at different heights above the solar limb. Therefore, the relevant radial profiles are very steep and kinetic temperature values are significantly larger than those inferred from the observations carried out in the subsequent days, when the increase in kinetic temperature is more gradual (see, for instance, the two streamers observed on Nov. 9,1997 ). Above $4 R_{\odot}$ the different kinetic temperature profiles spread out radially over a large interval of temperatures (ranging from about $4 \times 10^{6}$ up to $2 \times 10^{7} \mathrm{~K}$ ). Note the small decrease in the kinetic temperature for one of the streamers observed on Nov. 9. Substantial differences in O VI kinetic temperatures have been recognized previously between an equatorial and a mid-latitude streamer observed at different phases of the solar cycle by Frazin et al. (1999). The kinetic behaviour inferred in the present analysis from the observations of Nov. 3 and 5, 1997 seems to agree very closely with that reported by these authors for the equatorial streamer (also reported in Frazin et al. 2003), while our mid-latitude streamer regions observed on Nov. 6, 7 and 9, 1997 show kinetic properties intermediate between those reported by Frazin et al. (1999) for the equatorial and mid-latitude streamers.

The O VI adjacent regions observed on 1997 exhibit kinetic temperatures progressively higher with increasing height, from $2 R_{\odot}$ outwards, as expected for open-field line regions. As already pointed out in previous papers (e.g. Abbo \& Antonucci 2002), the same kinetic temperature values are reached at lower heights in regions adjacent to the streamers than in the streamers themselves. This can be easily recognized, for instance, by comparing the profiles referring to both the streamer and adjacent region observed on Nov. 5, 1997: the plasma outside the streamer reaches a value of about $2 \times 10^{7} \mathrm{~K}$ at $2.7 R_{\odot}$, while the same kinetic temperature characterizes the plasma inside the streamer at about $4.1 R_{\odot}$, so that the two radial profiles pratically overlap if the kinetic temperature curve referring to the streamer is shifited by about $1.4 R_{\odot}$ inwards.

The different $\mathrm{O}$ VI and Ly $\alpha$ kinetic temperature behaviour, both inside and outside streamers, may confirm previous claims for a heating mechanism that preferentially heats $\mathrm{O}$ VI ions in comparison with protons (e.g. Cranmer et al. 1999a; Frazin et al. 2003), more efficiently working in the regions surrounding the streamer structures where the magnetic field lines open. Incidentally, in this case the Ly $\alpha$ kinetic temperature might also provide a measure of the proton thermal temperature in the corona because the line seems only slightly influenced by nonthermal motions. Furthermore, the very steep gradient characterizing the $\mathrm{O}$ VI kinetic temperature profiles referring to the adjacent regions might also be considered as the typical signature of a possible energy deposition due to ion-cyclotron resonant dissipation of high-frequency Alfvén waves, whose spectrum very strongly steepens from the base of the corona to higher heliocentric distances (see, e.g., Cranmer et al. 1999b). The evidence for microscopic anisotropy in the O VI velocity distributions, with the component of the most probable speed perpendicular to the magnetic field greater than the parallel one, found in both open (e.g. Cranmer et al. 1999a) and closed (e.g. Frazin et al. 2003) coronal configurations, could provide additional support to this hypothesis.

The scenario concerning the data collected in 2000 is deeply different and limited to the OVI lines only. Within $2.0 R_{\odot}$, in-streamer regions observed on different days show almost identical kinetic temperatures. They abruptely steepen between 2.0 and $2.5 R_{\odot}$, increasing by a factor of $\simeq 3.5$, and then asymptotically reach a value of about $10^{7} \mathrm{~K}$ at $3.6 R_{\odot}$. The kinetic temperatures referring to the regions outside the streamers are about 2 times higher than the corresponding streamer values within $2 R_{\odot}$, then increase by a factor of 2 , between 2.5 and $2.8 R_{\odot}$, mixing with the streamer profiles at higher heights. Hence most of the difference in the kinetic conditions characterizing solar maximum streamers and adjacent regions appears to be confined within about $2 R_{\odot}$, while at greater distances from the Sun it may become difficult to discriminate between open and closed structures. This behaviour is opposite to that shown by solar minimum streamers and their surroundings, where the differences in kinetic temperature are quite negligible near the Sun and become progressively more significant at greater heliocentric distances.

For an easier comparison of the characteristics inferred for closed and open field-line regions at different phases of solar activity, we also derived mean radial profiles of the kinetic temperature representative of O VI streamers and adjacent regions at solar minimum and maximum, by averaging the corresponding individual curves reported in the top panels of Fig. 4. Due to the different behaviour of the in-streamer regions observed on Nov. 3 and 5, 1997 with respect to all the others (we postpone a discussion of the possible reasons for these differences to Sect. 4) we computed two different mean curves relevant for streamers observed in 1997, either including or not the observations carried out on Nov. 3 and 5, and then compared the mean kinetic temperature profile obtained for the streamers observed in 2000 with both of them. The 2000/1997 mean kinetic temperature ratios are reported in the righ-bottom panel of Fig. 4. Within $2.0 R_{\odot}$ the mean O VI kinetic temperatures referring to the streamer regions observed in 2000 are always lower than those referring to 1997. At greater heliocentric distance the mean kinetic temperatures referring to the streamers observed in 2000 remain systematically lower than the corresponding quantities computed by including all the streamer data collected in 1997 whereas, neglecting the observations of Nov. 3 and 5, 1997, the 2000 data approach the 1997 mean values around $2.5 R_{\odot}$ and exceed them above $3 R_{\odot}$. 


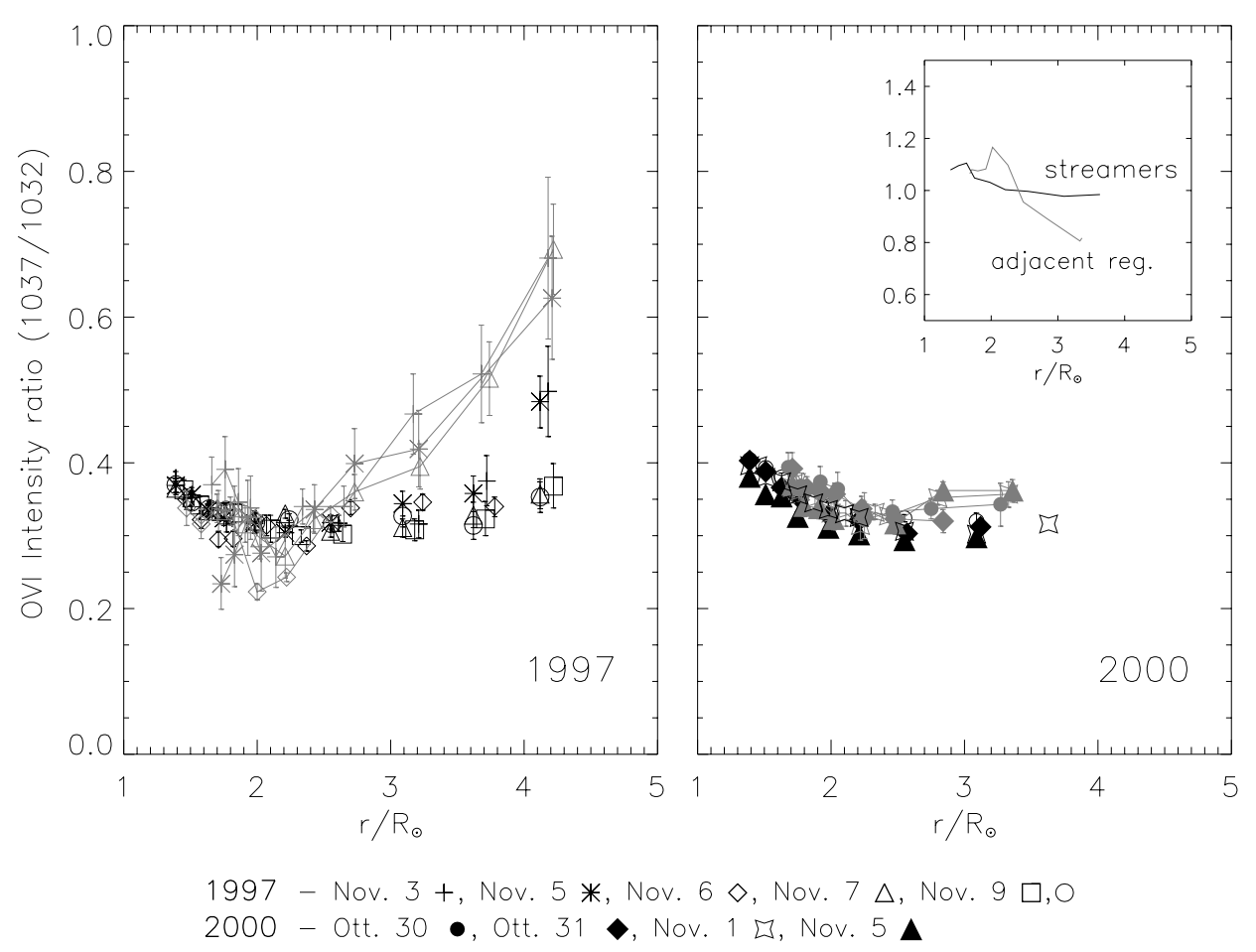

Fig. 5. As in Fig. 3, for the O VI (1037 ̊/1032 $\AA$ ) line ratio. The inset shows the comparison between the 2000 and 1997 O VI mean line ratios referring to streamers (black line) and adjacent regions (grey line), respectively.

At $1.7 R_{\odot}$, the mean kinetic temperature for the adjacent regions observed in 2000 is about 0.6 times lower than the corresponding mean value deduced from the 1997 data, then becomes even lower within $2.5 R_{\odot}$ and again slightly increases at greater heliocentric distances, reaching a value about 2.5 lower than that of the adjacent regions observed at solar minimum.

\subsection{Outflow velocity}

The OVI resonance doublet lines are a mixture of resonantly scattered and collisionally excited components. For lines excited only by resonant scattering the ratio of their intensities $(I(1037 \AA) / I(1032 \AA))$ is close to 0.25 , whereas for purely collisional excitation it is $\simeq 0.5$ (e.g. Noci et al. 1987). The relative contribution of these components strongly depends on outflow velocity through the progressive depletion, with increasing wind speed, of the radiative component, known as the Doppler dimming effect. Therefore, the intensity ratio of the O VI lines is a sensitive measure of the Doppler dimming, affecting them for outflow speeds from about $50 \mathrm{~km} \mathrm{~s}^{-1}$ to $400 \mathrm{~km} \mathrm{~s}^{-1}$, as proved by many authors (Noci et al. 1987; Dodero et al. 1998; Li et al. 1998; Cranmer et al. 1999a). Note that for outflow velocities greater than about $100 \mathrm{~km} \mathrm{~s}^{-1}$ the line ratio is $\geq 0.5$. Figure 5 reports the O VI line intensity ratio as a function of the heliocentric distance for the coronal regions observed in 1997 and 2000. Analogously to Figs. 3 and 4, the comparison between the 2000 and 1997 mean profiles for both streamers and adjacent regions is also reported. The uncertainties are those deduced from the photon counting statistics of the relevant line intensities. As in previous figures, the error bars are not reported whenever smaller than the symbol size. Within $\simeq 3.8 R_{\odot}$, the $\mathrm{O}$ VI intensity ratio obtained from the data collected in 1997 and referring to the streamer structures ranges between 0.3 and 0.4 . In particular, it is $\simeq 0.37$ both close to the solar limb and towards greater heights with a minimum of about 0.3 at $2 R_{\odot}$, as a consequence of the radial decrease in the electron density which makes the resonantly scattered component of the $\mathrm{O} V I$ lines contribute to the line intensities significantly more than the collisionally excited one (see, e.g., Noci et al. 1987). At greater heliocentric distances, i.e. between 3.8 and $4.2 R_{\odot}$, the $\mathrm{O} V \mathrm{VI}$ intensity ratio referring to the observations performed on Nov. 3 and 5, 1997 increases up to $\simeq 0.5$.

In the range of heliocentric distances $1.39-2.7 R_{\odot}$, the behaviour of the curves referring to the regions surrounding the streamers is very similar to that shown by the streamers themselves, except for the adjacent regions observed on November 3 and 6, 1997, which between 1.7 and $2.2 R_{\odot}$ exhibit O VI ratio values very close to the 0.25 limit for low-density or negligible outflow speed conditions (see, e.g., Noci et al. 1987). However, above $2.8 R_{\odot}$ all curves begin to increase rather abruptly with height and reach relatively high values (0.6-0.7).

According to Noci et al. (1987) and Dodero et al. (1998), these results might suggest that the outflow plasma velocity cannot exceed $\sim 50 \mathrm{~km} \mathrm{~s}^{-1}$ up to about $3 R_{\odot}$ in both streamers and adjacent regions. Above $3 R_{\odot}$, the outflow velocity remains below $50 \mathrm{~km} \mathrm{~s}^{-1}$ in all the observed in-streamer regions at any heliocentric distance, except inside the streamer observed on Nov. 3 and 5, 1997, where an outflow of about $100 \mathrm{~km} \mathrm{~s}^{-1}$ can be inferred, with most of the acceleration occurring between 3.6 and $4.1 R_{\odot}$. The result concerning the observations of Nov. 3 and 5, 1997 is consistent with that also found by Strachan et al. (2002) for the axis of an equatorial 
streamer at solar minimum. If the transition from no detectable outflow to speeds as high as $100 \mathrm{~km} \mathrm{~s}^{-1}$ along the axis of a coronal streamer can be attributed to the transition from closed to open field lines, i.e. the streamer cusp (cf. Strachan et al. 2002), the evidence of no transition to significant flows in the studied range of heliocentric distances for many of the streamer regions scanned in 1997 might simply suggest that their cusps could be located above $4.1 R_{\odot}$. Moreover, the results reported by Frazin et al. (2003) for the equatorial streamer observed on October 1996 do not support the idea of flows inside the streamer faster than about $50 \mathrm{~km} \mathrm{~s}^{-1}$ up to $4.1 R_{\odot}$. An upper limit of about $105 \mathrm{~km} \mathrm{~s}^{-1}$ was only derived by these authors at $5.1 R_{\odot}$. Evidence for fast outflows can be deduced for the regions adjacent to the solar minimum streamers in the range of heliocentric distance 3-4 $R_{\odot}$, with values probably higher than $200 \mathrm{~km} \mathrm{~s}^{-1}$ at $4 R_{\odot}$ (see, e.g., Dodero et al. 1998).

The OVI ratio profiles referring to the data collected in 2000 are very probably the consequence of different coronal dynamical conditions in comparison with the solar minimum situation. In- and out-of-streamer regions exhibit practically the same trend with all the O VI intensity ratio profiles always below or equal to 0.4 . In particular, the ratio is 0.4 at about $1.39 R_{\odot}$, then shows a minimum of about 0.28 at $2.5 R_{\odot}$ and a hint of increasing weakly again towards greater heliocentric distances, a little bit more pronounced in regions adjacent to the streamers than in the streamers themselves. An outflow velocity below $50 \mathrm{~km} \mathrm{~s}^{-1}$ can be inferred everywhere from these results. Unfortunately, our data do not allow us to check whether the observed weakly increasing trend in the outflow speed outside streamers beginning at about $3.1 R_{\odot}$ persists at larger heliocentric distances.

The mean behaviour deduced for individual streamer and adjacent region data collected in 1997, compared to that referring to the corresponding regions observed in 2000, helps to show that streamers observed at solar minimum and solar maximum show very similar characteristics from a dynamical point of view. Conversely, the adjacent regions observed at solar maximum appear to be quite stationary also at heliocentric distances larger than 3-4 $R_{\odot}$, where a transition from stationary conditions to detectable flows seems to occur in the adjacent regions observed at solar minimum.

\subsection{Electron density}

The UVCS pB measurement performed with its WLC refers within $5 R_{\odot}-$ to the visible polarized brightness of the $\mathrm{K}$ corona, i.e. the white-light coronal component coming from the Thompson scattering of photospheric light by free electrons (e.g. Billings 1966). It can be directly related to the electron density integrated over the line-of-sight, with no dependence on other plasma parameters. The computation of the local electron density requires, however, a detailed model of the coronal structures unless a spherical symmetry is adopted. In this case the local electron density can be derived analytically through Van de Hulst's inversion technique (Van de Hulst 1950). This is also the assumption we adopted to derive electron densities for the streamers and adjacent regions observed in 1997 and 2000.
The results are reported in Fig. 6 as a function of the heliocentric distance. Slit heights below $1.7 R_{\odot}$ have been neglected, because there the stray light level in the visible light channel is too high for a meaningful determination of $\mathrm{pB}$ (see Romoli et al. 2002). Each point refers to a 14 arcsec $\times 14$ arcsec region approximately corresponding to the central bins of the UVCS entrance slit that during the daily scans looked at portions of the solar image either inside or outside the observed streamers. In Fig. 6 the electron density values inferred for streamers and adjacent regions are reported in different panels for clarity. The error bars have been computed by the photon counting statistics of the $\mathrm{pB}$ data and are not reported whenever smaller than the symbol size.

The most evident feature of the electron density radial profiles referring to solar minimum streamers and surrounding regions is a systematic tendency for streamers to be denser than their surroundings, as expected. In addition, for the data collected on Nov. 7, 1997, characterized by a good overlapping of the visible light channel pinhole on the streamer axis at each scan position, we can identify the streamer core as the place inside the structure where the electron density has a maximum. This result is in good agreement with that recently found by Abbo \& Antonucci (2002), Strachan et al. (2002), Frazin et al. (2003) and Spadaro et al. (2004). Moreover, our data concerning solar minimum streamer regions well reproduce the electron density values reported by Abbo \& Antonucci (2002), empirically derived from the ratio of the collisional to radiative component of the O VI $1032 \AA$ line for a static streamer, while those obtained by Strachan et al. (2002) and Frazin et al. (2003) from LASCO-C2 white light images for the central part of two equatorial streamers at solar minimum are higher at any heliocentric distance than the data deduced for the axis of the streamer observed on Nov. 7, 1997. This holds even taking into account the difference in the $\mathrm{pB}$ calibrations of LASCO-C2 and UVCS WLC of about 20\% (see Frazin et al. 2002). The present data concerning adjacent regions at solar minimum are also in good agreement with those reported by Abbo \& Antonucci (2002) for streamer surroundings and deduced in the hypothesis of an expanding corona.

The electron density values inferred from the data collected in 2000 refer only to streamer regions. Individual profiles show a very similar trend. The transition between the solar minimum to maximum seems to be characterized by a global increase of the electron density inside streamers that is more pronounced at low heliocentric distance, as can be noted by comparing the mean electron densities obtained on the basis of all the streamers observed in 2000 and 1997. At low heliocentric distances $\left(1.7 R_{\odot}\right)$ solar maximum streamers are on average almost 4 times denser than their solar minimum counterparts. The difference between the electron densities inside streamers observed at solar minimum and inside those observed at solar maximum progressively decreases with increasing distance, the two values being almost comparable at $3.6 R_{\odot}$. The more rapid decline of the electron density in solar maximum streamers with respect to solar minimum might suggest the presence during the phase of maximum activity of denser and spatially more compact closed field-line structures, which radially protrude less into the extended corona. This suggestion seems to 


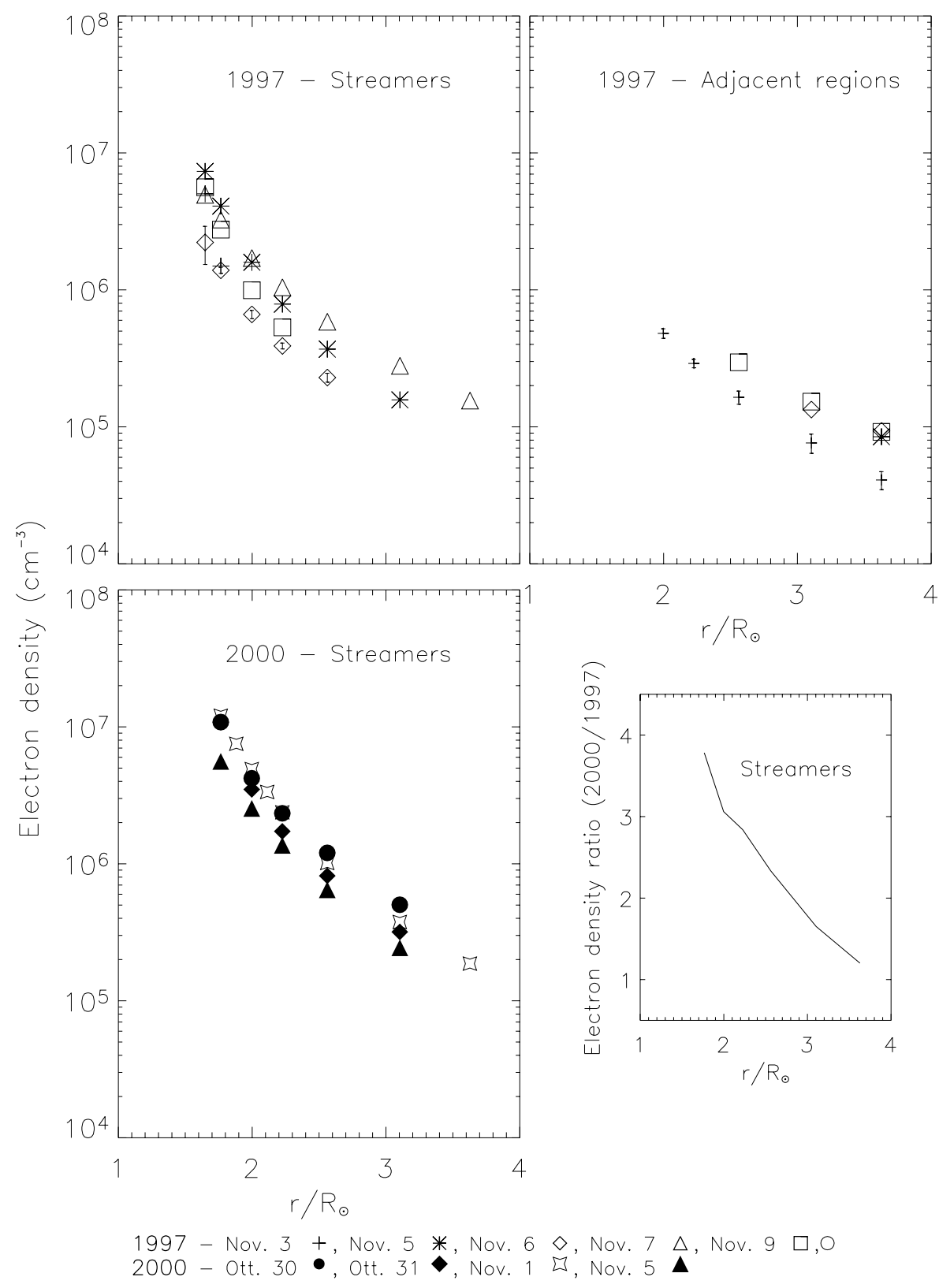

Fig. 6. Radial profiles of electron density inferred for streamers (top-left panel) and adjacent region (top-right panel) from UVCS pB measurements collected in 1997 and for streamers only (bottom-left panel) in 2000. Bottom-right panel: 2000/1997 electron density ratio as referring to streamer regions, obtained by averaging the individual profiles of the relevant structures at solar maximum and solar minimum, respectively.

be supported, in particular, by the LASCO white-light images shown in Fig. 1.

\section{Conclusions}

The most significant results of our analysis, which compares in- and out-of-streamer physical conditions deduced from SOHO/UVCS observations carried out during different phases of solar activity cycle, deserve further comment.

The mid-latitude in-streamer region observed for a period of four days in 1997 showed an interesting temporal evolution in O VI kinetic and dynamical plasma properties. On Nov. 3 and 5, 1997 the radial profiles of the perpendicular kinetic temperature abruptly steepen within a few tenths of a solar radius. Consequently, these temperatures are significantly larger than those characterizing the same region on subsequent days, as well as the other two streamers observed on Nov. 9, 1997. Moreover, a particular behaviour in the O VI doublet intensity ratio also characterizes the same in-streamer region observed in Nov. 3 and 5, 1997 with respect to all the other solar-minimum streamers, with a sharp transition towards high outflow speed regimes between 3.6 and $4.1 R_{\odot}$ and a probable outflow velocity of about $100 \mathrm{~km} \mathrm{~s}^{-1}$ at $4.1 R_{\odot}$. However, the enhancements in perpendicular kinetic temperature and O VI doublet ratio are not cospatial, so that it can be quite reasonably conjectured that the same mechanism, capable of heating the plasma already at low heliocentric distances, might also progressively accelerate the solar wind to reach detectable 
velocity values only at fairly large heliocentric distances, probably where the transition from closed to open field lines takes place. A similar spatial delay in the kinetic and dynamical plasma responses has been detected in latitude by Spadaro et al. (2004) in their analysis of plasma parameters inside and outside mid-latitude streamers at solar minimum.

This particular behaviour, formally reminiscent of the typical energetic response of the coronal plasma to the ioncyclotron resonant dissipation of high-frequency Alfvén waves (see, e.g., Cranmer et al. 1999b) commonly observed in open field line configurations (and in our solar minimum adjacent regions, as well), raises a crucial question: is there day by day a real temporal evolution in the plasma conditions of the observed streamer region (arising from a complex and extremely variable ensemble of underlying loops, clearly visible in the images taken by SOHO/EIT at the same time), or are the observed differences in the streamer properties simply due to different projections along the line-of-sight of the same streamer region dragged westward by the solar rotation? In the latter hypothesis we should observe a variable mix of in-streamer and out-of streamer material consequently exhibiting intermediate characteristics between streamers and adjacent region conditions. In the first case, conversely, the significant differences in kinetic temperature deduced from the observations could be attributed to an effective change over time in the conditions of the observed streamer region. At this point it would be interesting to investigate whether the heating mechanisms at work inside and outside streamers are of different nature or the different magnetic configuration is responsible for the different response. A variety of observational pieces of evidence are making this question currently more and more frequently addressed (see, e.g., Strachan et al. 2002; Frazin et al. 2003), but not yet completely answered.

The results obtained from the analysis of the data collected in 2000 reveal impressive changes in the global appearence of the solar corona. In fact the solar maximum streamers, up to 4 times brighter than the streamers observed at solar minimum, appear embedded in background surroundings on average more than one order of magnitude brighter in the EUV emission than at solar minimum. On the other hand, we cannot rule out contamination effects from closed structures along the line-of-sight that can enhance the emission in regions adjacent to streamers.

Strong evidence for quite similar plasma conditions inside and outside coronal streamers at solar maximum comes from the analysis of their kinetic and dynamical behaviour, making difficult any attempt to characterize the solar maximum different magnetic configurations through empirical parameters. This is surprisingly true also for the observations carried out on Nov. 5, 2000, when the UVCS slit bridged a streamer region and an equatorial coronal hole, well visible in the LASCO C2 white-light image of that day (see Fig. 1). In this case line-ofsight contamination effects might be ruled out. Sharp different signatures in the perpendicular kinetic temperature of streamers and adjacent regions have been detected in the present analysis only within $2 R_{\odot}$, where in- and out-of-streamer values differ by about a factor of 2, showing the adjacent regions with higher kinetic temperatures. Moreover, neither of the kinetic temperature radial profiles obtained at solar maximum inside or outside streamers show the typical behaviour, exhibited predominantly by solar minimum adjacent regions, of a heating mechanism probably due to wave dissipation. In addition, the $\mathrm{O}$ VI doublet intensity ratio profiles inferred for open and closed magnetic field line configurations at solar maximum are compatible with plasma conditions essentially stationary both inside and outside streamers at any heliocentric distances. This is a striking difference with respect to the behaviour of the solar minimum adjacent regions, where velocities of $200 \mathrm{~km} \mathrm{~s}^{-1}$ and higher may be inferred at heliocentric distances around $4 R_{\odot}$. The transition between the minimum and maximum seems to be characterized by an increase of the electron density inside streamers, that is more pronounced at low heliocentric distance, while higher in the corona these differences progressively flatten. This occurrence might reveal a much more compact structure for solar maximum streamers, as well as a probable smaller extension of streamers into the solar wind, in comparison to solar minimum.

It is reasonable to believe that as the solar cycle progresses from solar minimum to solar maximum, besides the global magnetic structure, the small-scale magnetic field topology also consequently changes, as well as the morphology of streamers (e.g., the latitudinal extent of their base and the height of their cusps), as do the plasma properties inside and outside streamers. Accordingly, the coronal plasma response to coronal heating and acceleration mechanisms can possibly be modified. Strong supports for this idea come from Ulysses measurements which pointed out very strong changes in the solar wind structure at solar maximum (Smith et al. 2003): although very probably during a short-lived phase around solar maximum, fast solar wind may be absent and variable slow wind may dominate at all latitudes (see, for instance, Fig. 2 in Smith et al. 2003). For this reason it would be of great interest to investigate theoretically how the heating and acceleration processes currently proposed to explain solar minimum properties critically depend on changes in the ambient conditions. In this context the results of the analysis of different coronal structures both at solar minimum and maximum, presented in this work, might contribute to test quantitatively different theories for solar wind acceleration and heating, and probably to ascertain if our capability of modelling the solar corona at maximum is currently sufficient to explain the observational data or if some more modelling is necessary.

Neither of our mid-latitude streamers both at solar minimum (as those also monitored by Spadaro et al. 2004) and solar maximum (see, e.g., Bemporad et al. 2003) observed in the OVI line show the same bifurcated configuration reported by other authors (e.g. Noci et al. 1997; Raymond et al. 1997; Dobrzycka et al. 1999; Zangrilli et al. 1999; Strachan et al. 2002; Frazin et al. 2003) for typical equatorial helmetstreamers at solar minimum. This fact should confirm some previous claims of intrinsic differences between mid-latitude and equatorial streamers (e.g. Noci et al. 1997)

Acknowledgements. This work was made possible by the UVCS/SOHO instrument and Flight Operations team. The UVCS is the result of a collaborative effort between NASA and the Agenzia Spaziale Italiana (ASI), with a Swiss participation. R. Ventura and 
D. Spadaro wish to thank all the staff of the MEDOC center at the Institut d'Astrophysique Spatiale in Orsay - Paris for kind hospitality. The authors also wish to thank R. Suleiman for his help in the preliminary reduction of the UVCS raw data. They also thank J. Raymond for useful comments and suggestions which contributed to improve the paper. This work was supported in part by the Agenzia Spaziale Italiana (contract ASI I/R/84/02).

\section{References}

Abbo, L., \& Antonucci, E. 2002, in SOHO 11 Symp., From Solar Minimum to Max: Half a Solar Cycle with SOHO, ed. A. Wilson (Noordiwijk: ESA), ESA-SP, 508, 477

Antonucci, E., Giordano, S., Benna, C., et al. 1997, in Fifth SOHO Workshop, The Corona and Solar Wind near Minimum Activity, ed. A. Wilson (Noordwijk: ESA), ESA-SP 404, 175

Banerjee, D., Teriaca, L., Doyle, J. G., \& Lemaire, P. 2000, Sol. Phys., 194, 43

Bemporad, A., Poletto, G., Suess, S. T., et al. 2003, ApJ, 593, 1146

Beckers, J. M., \& Chipman, E. 1974, SoPh, 34, 151

Billings, D. E. 1966, A Guide to the Solar Corona (New York: Academic Press)

Brueckner, G. E., Howard, R. A., Koomen, M. J., et al. 1995, Sol. Phys., 162, 357

Cranmer, S. R., Kohl, J. L., Noci, G., et al. 1999a, ApJ, 511, 481

Cranmer, S. R., Field, G. B., \& Kohl, J. L. 1999b, ApJ, 518, 937

Delaboudinière, J.-P., Artzner, G. E., Brunaud, J., et al. 1995, Sol. Phys., 162, 291

Dodero, M. A., Antonucci, E., Giordano, S., \& Martin, R. 1998, Sol. Phys., 183, 77

Domingo, V., Fleck, B., \& Poland, A. I. 1995, Sol. Phys., 162, 1

Dobrzycka, D., Cranmer, S. R., Panasyuk, A. V., Strachan, L., \& Kohl, J. L. 1999, J. Geophys. Res., 104, 9791

Fineschi, S., Gardner, L. D., Kohl, J. L., Romoli, M., \& Noci, G. C. 1998, Proc. SPIE, 3443, 67

Frazin, R. A., Ciaravella, A., Dennis, E., et al. 1999, Space Sci. Rev., 87, 189

Frazin, R. A., Romoli, M., Kohl, J. J., et al. 2002, in The Radiometric Calibration of SOHO, ed. A. Pauluhn, M. C. E. Huber, R. von Steiger (Noordwijk: ESA), ISSI SR-002, 249

Frazin, R. A., Cranmer, S. R., \& Kohl, J. L. 2003, ApJ, 597, 1145

Gabriel, A. H., Bely-Dubau, F., \& Lemaire, P. 2003, ApJ, 289, 623

Gardner, L. D., Kohl, J. L., Daigneau, P. S., et al. 1996, Proc. SPIE, 2831, 2

Giordano, S., Antonucci, E., Noci, G., Romoli, M., \& Kohl, J. L. 2000, ApJ, 531, L79
Habbal, S. R., Woo, R., Fineschi, S., et al. 1997, ApJ, 489, L103

Hassler, D. M., Dammasch, I. E., Lemaire, P., et al. 1999, Science, 283,810

Hyder, C. L., \& Lites, B. W. 1970, Sol. Phys., 14, 147

Kohl, J. L., Esser, R., Gardner, L. D., et al. 1995, Sol. Phys., 162, 313

Kohl, J. L., Noci, G., Antonucci, E., et al. 1997, Sol. Phys., 175, 613

Kohl, J. L., Noci, G., Antonucci, E., et al. 1998, ApJ, 501, L127

Li, X., Habbal, S. R., Kohl, J. L., \& Noci, G. 1998, ApJ, 501, L133

Low, B. C. 2001, J. Geophys. Res., 106, 25141

McComas, D. J., Elliott, H. A., Gosling, J. T., et al. 2002, GeoRL, 29, 4

McComas, D. J.,Elliott, H. A., \& von Steiger, R. 2002, GeoRL, 29, 28 Noci, G., Kohl, J. L., \& Withbroe, G. L. 1987, ApJ, 315, 706

Noci, G., Kohl, J. L., Antonucci, E., et al. 1997, in Fifth SOHO Workshop, The Corona and Solar Wind near Minimum Activity, ed. A. Wilson (Noordwijk: ESA), ESA-SP 404, 75

Ofman, L. 2000, Geophys. Res. Lett., 27, 2885

Patsourakos, S., \& Vial, J.-C. 2000, A\&A, 359, L1

Phillips, J. L., Bame, S. J., Barnes, A., et al. 1995, Geophys. Res. Lett., 22, 3301

Raymond, J. C., Kohl, J. L., Noci, G., et al. 1997, Sol. Phys., 175, 645

Romoli, M., Benna, C., Cranmer, et al. 1997, in Fifth SOHO Workshop, The Corona and Solar Wind Near Minimum Activity, ed. A. Wilson, (Noordwijk: ESA), ESA-SP 404, 633

Romoli, M., Frazin, R. A., Kohl, J. L., et al. 2002, in The Radiometric Calibration of SOHO, ed. A. Pauluhn, M. C. E. Huber, R. von Steiger (Noordwijk: ESA), ISSI SR-002, 181

Sheeley, N. R., Jr., Wang, Y.-M., Hawley, S. H., et al. 1997, ApJ, 484, 472

Smith, E. J., Marsden, R. G., Balogh, A., et al. 2003, Science, 302, 1165

Spadaro, D., Ventura, R., Cimino, G., \& Romoli, M. 2004, A\&A, in press

Strachan, L., Panasyuk, A. V., Dobrzycka, D., et al. 2000, J. Geophys. Res., 105, 2345

Strachan, L., Suleiman, R., Panasyuk, A. V., Biesecker, D. A., \& Kohl, J. L. 2002, ApJ, 571, 1008

Teriaca, L., Poletto, G., Romoli, M., \& Biesecker, D. A. 2003, ApJ, 588, 566

Van de Hulst, H. C. 1950, Bull. Astron. Inst. Netherlands, 11, 135

Wang, Y.-M. 1994, ApJ, 435, L153

Zangrilli, L., Nicolosi, P., Poletto, G., et al. 1999, A\&A, 342, 592

Zangrilli, L., Poletto, G., Nicolosi, P., Noci, G., \& Romoli, M. 2002, ApJ, 574, 477 